\title{
INDICATOR SPECIES POPULATION MONITORING IN ANTARCTICA WITH UAV
}

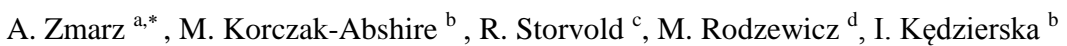 \\ ${ }^{a}$ Depart. of Geoinformatics, Cartography and Remote Sensing, University of Warsaw, Faculty of Geography and Regional Studies, \\ Krakowskie Przedmieście 30, 00-927 Warsaw, Poland - azmarz@uw.edu.pl \\ ${ }^{b}$ Depart. of Antarctic Biology, Institute of Biochemistry and Biophysics Polish Academy of Sciences, Ustrzycka 10/12, 02-141 \\ Warsaw, Poland - mka@ibb.waw.pl \\ ${ }^{c}$ Northern Research Institute Tromsø, P.O.Box 6434, 9294 Tromsø, Norway - rune.storvold@ norut.no \\ ${ }^{\mathrm{d}}$ Institute of Aeronautics and Applied Mechanics, Warsaw University of Technology, Nowowiejska 24, 00-665 Warsaw, Poland- \\ miro@meil.pw.edu.pl
}

KEY WORDS: Indicator species, penguins, monitoring, Antarctica, UAV.

\begin{abstract}
:
A program to monitor bird and pinniped species in the vicinity of Arctowski Station, King George Island, South Shetlands, Antarctica, has been conducted over the past 38 years. Annual monitoring of these indicator species includes estimations of breeding population sizes of three Pygoscelis penguin species: Adélie, gentoo and chinstrap. Six penguin colonies situated on the western shores of two bays: Admiralty and King George are investigated.

To study changes in penguin populations Unmanned Aerial Vehicles were used for the first time in the 2014/15 austral summer season. During photogrammetric flights the high-resolution images of eight penguin breeding colonies were taken. Obtained high resolution images were used for estimation of breeding population size and compared with the results of measurements taken at the same time from the ground. During this Antarctic expedition eight successful photogrammetry missions (total distance $1500 \mathrm{~km}$ ) were performed. Images were taken with digital SLR Canon 700D, Nikon D5300, Nikon D5100 with a 35mm objective lens. Flights altitude at 350 - 400 AGL, allowed images to be taken with a resolution GSD (ground sample distance) less than $5 \mathrm{~cm}$. The Image J software analysis method was tested to provide automatic population estimates from obtained images. The use of UAV for monitoring of indicator species, enabled data acquisition from areas inaccessible by ground methods.
\end{abstract}

\section{INTRODUCTION}

\subsection{Project MONICA}

In the Antarctic summer season 2014/2015 Polish-Norwegian project: "A novel approach is the monitoring of the impact of climate change on Antarctic ecosystems - MONICA" was started. The main goal of this project is to study the impact of climate change on Antarctic ecosystem and biodiversity. Data presented here is an important contribution to the monitoring of the ecosystem as recommended by the Convention for the Conservation of Antarctic Marine Living Resources (CCAMLR). Two UAVs (PW-ZOOM, CryoWing) specially designed to work in polar conditions were used in order to perform the tasks of estimating breeding population size of indicator species (Korczak-Abshire, 2014).

\section{AIRCRAFT AND INSTRUMENTATION}

\subsection{PW-ZOOM Unmanned Photogrammetric Aircraft}

A special UAV, which is able to carry a high-resolution camera and to make autonomous photogrammetry missions was designed by the WUT partner of consortium (Hajduk, 2014). The plane was named PW-ZOOM (Figure1). Basic data of the plane: wing span $-3,5$ meters, fuselage length $-1,7 \mathrm{~m}$, take-off weight $23 \mathrm{~kg}$, cruising speed $115 \mathrm{~km} / \mathrm{h}$, flying range $-170 \mathrm{~km}$. Apart from photogrammetry equipment:
- Canon 700D, digital SLR camera with Canon 35mm f/2.0 objective lens

- Nikon D5300 digital SLR camera with a Nikon 35mm $\mathrm{f} / 2.0$ objective lens

the PW-ZOOM was equipped with radio-control system, autopilot (Micropilot 2128g), telemetry modems (SATELLINE YM 6500), the recovery parachute system.

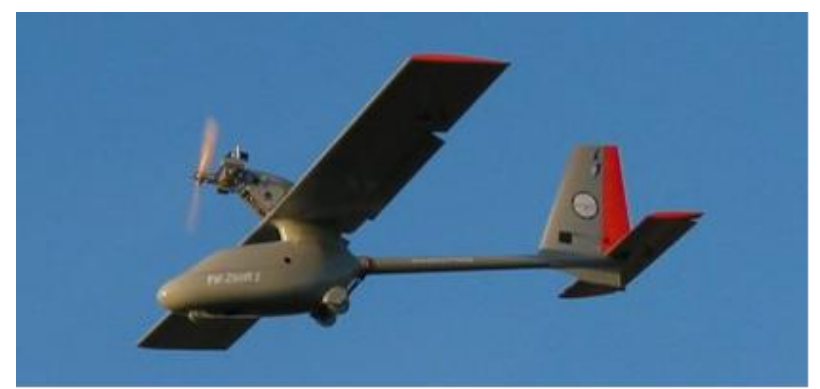

Figure 1. PW-ZOOM.

As the stability of flight is of crucial importance in photogrammetry missions, the PW-ZOOM designers focused their attention on two main factors influencing this feature: static \& dynamic stability of the plane, and a proper integration of the autopilot with the airplane dynamic systems. Outstanding airplane stability is achieved by mixing the "classic" lay-out of an aircraft with the high suspended wings (placed on a highpylon, which gives a self-stabilizing "pendulum effect"). The resistance to the wind gusts and turbulence, was reached by

\footnotetext{
Corresponding author
} 
optimization of aerodynamic characteristics following from the appropriate combination of geometric parameters, wing-loading and airfoil applied in the wings.

An integration of the autopilot with the airplane means much more than simple installing the autopilot on the airplane board, because this process mainly consists of proper setting of the autopilot parameters responsible for stabilization function and the parameters responsible for navigation function as well. The dynamic properties of the plane were taken into account as well, especially: cruising speed, turn radius, time necessary for reversion of the bank angle when turning direction has to be changed, increase of power consumption necessary for passing from the straight flight to turning at a fixed altitude, etc. Another factor considered by the authors were the control laws and navigation algorithms applied in the autopilot. The integration process was initially based on calculations, but final adjustment of all autopilot parameters was verified in an experimental way prior to the Antarctic expedition.

\subsection{CryoWing Unmanned Aircraft}

The CryoWing UAS (Solbø, 2013) was designed as a platform to carry science payloads for research within meteorology, climate, environment and Earth observation disciplines. The CryoWing (Figure 2) can carry up to $10 \mathrm{~kg}$ payload, and has a maximum range and endurance of $500 \mathrm{~km}$ and 5 hours, respectively. Though the complete CryoWing system is limited to Norut's (and partner institutions) in-house use, the system is assembled mainly from off-the-shelf components, and third party manufacturers utilize the same airframe in turn-key solutions (more information on web site, http://uas.norut.no).

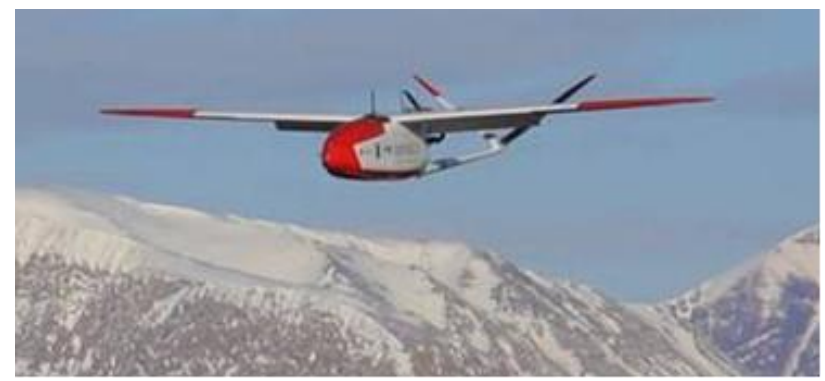

Figure 2. The CryoWing UAS.

The CryoWing can carry a wide variety of payload configurations. The core of the payload system is a computer for instrument control, data storage, data time stamping and communication routing. This payload computer, which runs a linux OS, is tightly connected to the power supply module that provides power to the system. This yields the opportunity to monitor the current consumption for each component, and the different instruments can be powered up and down when needed. Experience has shown that the ability to power cycle instruments can be essential to ensure measurements through long flights, especially for consumer grade cameras that are neither designed for automated operations, nor continuous measurements through several hours.

The operator on the ground is provided control and status for each instrument through a web interface on the ground station. In addition, if the required bandwidth is available, sample data can be downloaded in near real time.

In theory, all communication channels or modems that are supported by Linux can be utilized. For the King George Island flights discussed here, A $868 \mathrm{MHz}$ radio modem was utilized when within radio line of sight, with a fallback to Iridium satellite modem. The radio link is shared between flight control and payload communication, with flight control data prioritized. In addition there is a separate $2.4 \mathrm{GHz}$ link for manual control of the aircraft, used for launching and landing.

For this experiment the payload consisted of the following instrumentation:

- XsensMTi-G Inertial measurement and GPS unit (Xsens Technologies B.V., 2013).

- ICI 7640 LWIR camera (Infrared Cameras Inc., 2012)

- Nikon D5100 digital SLR camera with a Nikon 35mm f/1.8 objective lens.

The IMU was sampled at $100 \mathrm{~Hz}$, whereas the GPS receiver at $4 \mathrm{~Hz}$ and the IR camera at $1 \mathrm{~Hz}$, respectively. The Nikon D5100 shot images roughly every 2 seconds, the frequency of image acquisition is limited by the bandwidth of the USB bus that transfers the images to the camera.

\section{FLIGHT PLAN}

Flights have been designed over two protected areas: ASPA 128 (Admiralty Bay) and ASPA 151 (King George Bay), as well as Chabrier Rock Island (Figure 3). Creation of a flight plan was dependent on the size of the area, the atmospheric conditions on the day of the flight and the flight characteristics of the UAV. The target area size determines the manner in which the flight is planned and was related to the technical abilities of the UAV.

Depending on the actual size of the target, the image-taking operation were realized using a single flight or were divided into a number of related flights due to the target's shape and the UAV abilities (e.g. maximum flight length) and the safety rules. Flights performed over ASPA 128 covered $30 \%$ of its total polygon $(18.04 \mathrm{~km} 2)$ and over the entire surface of ASPA 151 $(1.32 \mathrm{~km} 2)$.

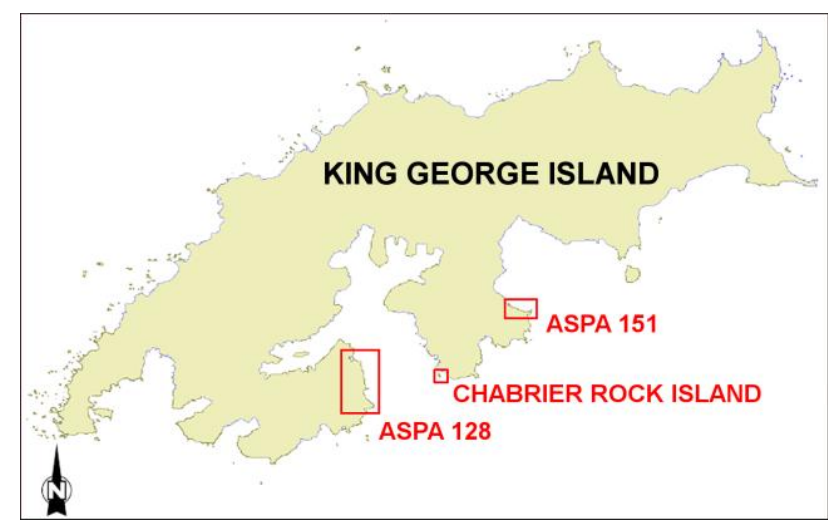

Figure 3. Investigated areas: Antarctic Specially Protected Areas (ASPA) no.128 and 151, as well as Chabrier Rock Island.

\section{GROUND CENSUS}

Ground censuses of penguin nests, taken at the same time as UAV missions, followed the standards defined by the CCAMLR Ecosystem Monitoring Program (CEMP standard methods, 2014). The breeding chronology of each species was a crucial factor (Figure 4). Ground census include selected: three 
breeding colonies of chinstrap penguin and three mixed colonies in ASPA 128 (Chwedorzewska and Korczak 2010) and ASPA 151 (Korczak-Abshire et al. 2012). In all mixed colonies all three species were nesting and occupying the same niche. In these six selected colonies one week after the peak of egg-laying the number of occupied nests were counted by three independent observers. Three separate counts were done of each of the breeding group in the colony on the same day. If one of the three counts differs more than $10 \%$ from the others, a fourth was made on the same day as the other three counts. If the results differ no more then by $10 \%$, the mean value was calculated. These mean values were compared with results of photo interpretation of obtained UAV images counted manually and automatically for selected groups.

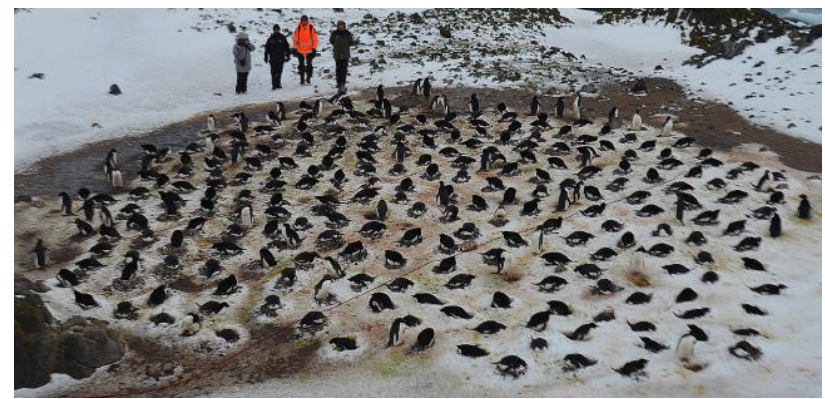

Figure 4. Ground censuses of penguin nests according to CEMP standard methods.

\section{IMAGE ANALYSIS}

Precision estimation of individual nests count was based on the photo interpretation of obtained UAV images of ASPA 151 penguin colony, particularly three selected breeding groups of Adélie penguin species. Small, medium and large in size breeding groups were chosen for this analysis (Figure 6). To automatically estimate the size of the colony of penguins, used images in JPEG format. The JPEG images were analysed using the Image $\mathrm{J}$ software (version 1.43q). A region of interest polygon was drawn over each penguin colony. The images in RGB (Red, Green, Blue) colour was converted to 8-bit images and adjusted the brightness and contrast (Figure 7). To automatically estimate the objects in the images uses two variables: the pixel size and circularity. Pixel size has been fixed at $10-11$, this means that the software automatically reject objects that contain smaller number of pixels (Figure 8). Circularity determines how the shape of object is close to the circle, to analysis set value of $0.5-0.7$, where 1 is circle.

\section{RESULTS AND CONCLUSIONS}

The Polish-Norwegian team did 8 flights with a total distance of about $1500 \mathrm{~km}$, in the time frame from 11 Nov to 6 Dec 2014. The image data was captured from an altitude of $350-400$ meters above sea level and covered the coast lines and hatching areas along the coasts of King George Island. The ground resolution for the visible camera was approximately $5 \mathrm{~cm}$ (Figure 5) and infrared camera $40 \mathrm{~cm}$.

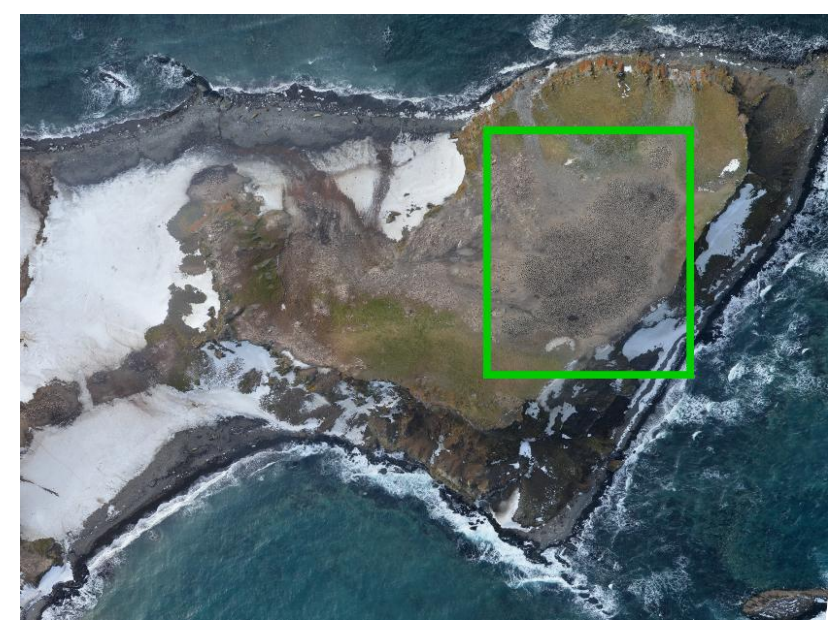

Figure 5. The image of ASPA 151 penguin colony (selected polygon framed in green), an example of results.

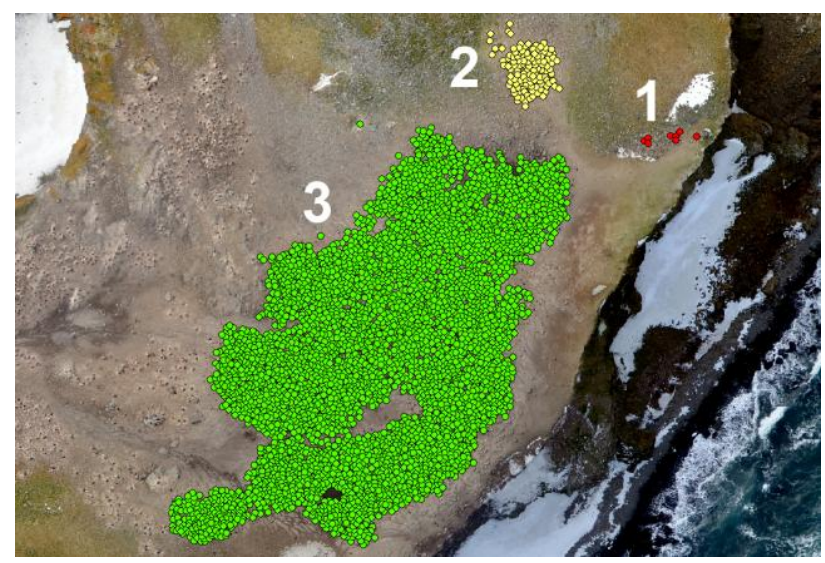

Figure 6. Adélie penguin breeding groups selected for comparison of manual and automatic estimation (ArcMap 10.1).

The imagery was taken with sufficient overlap to create orthorectified image mosaics as well as 3D models of the images using Agisoft Photoscan software. Images of this GSD allowed to locate and estimate the number of nests of Adélie, gentoo and chinstrap penguins. From the images more than 25 thousand penguin nests in eight breeding colonies located at Admiralty and King George Bays were counted (ASPA 128, ASPA 151, Chabrier Rock Island). Similar results were obtained for ground censuses of penguin nests according to CEMP standard methods. Finally, the result of automatic estimation of penguins did not differ by more than $10 \%$ from results obtained for ground censuses. 

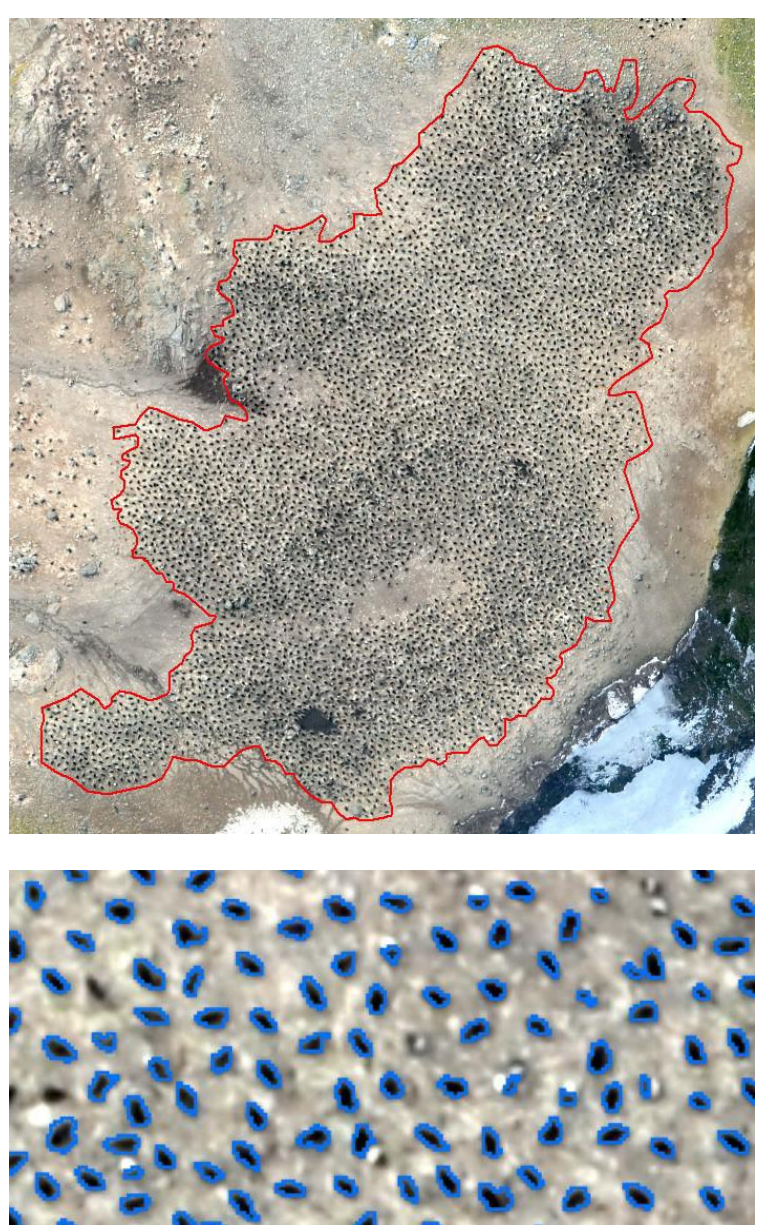

Figure 7. The polygon of the selected penguin group and result of automatic estimation of the objects.
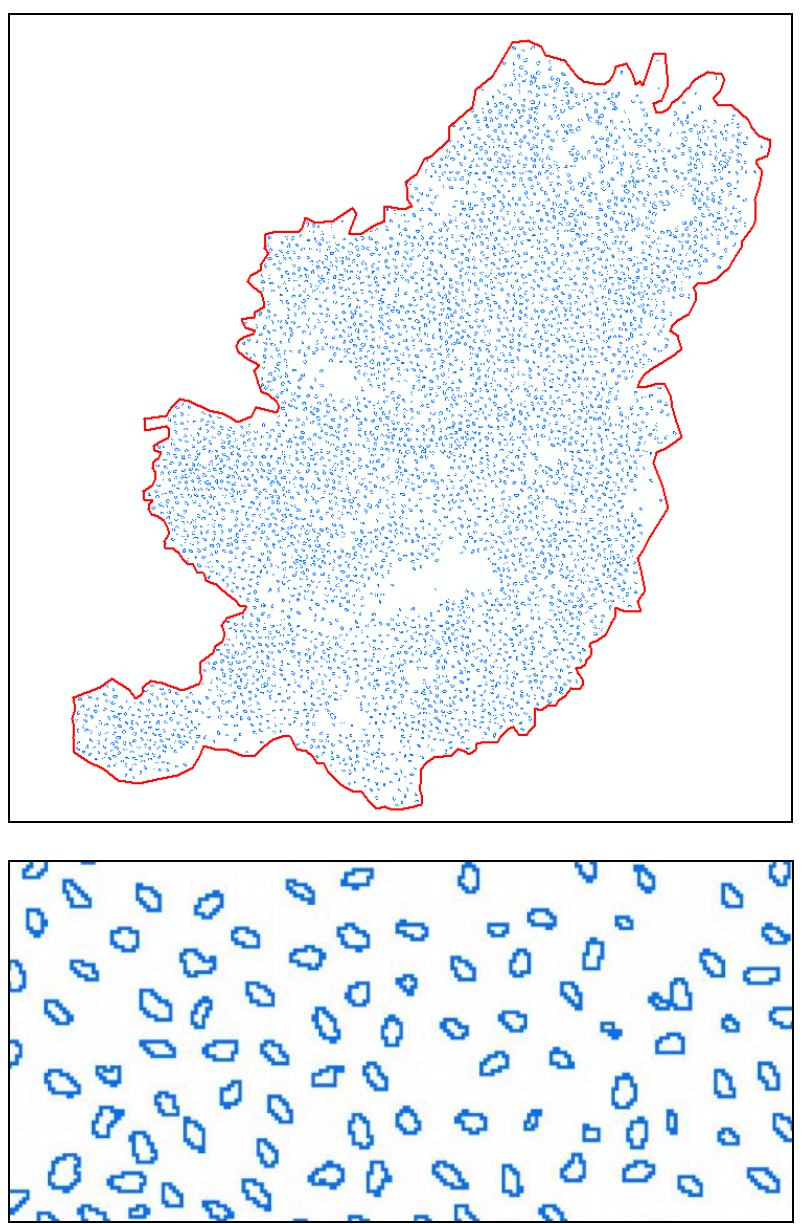

Figure 8. The result of automatic estimation of the counts of objects (penguins) (Image J).

In the next breeding season the extension of the research area and reaching out to the colonies that have so far not been accessible from the ground is planned. The use of UAV for purpose of CEMP monitoring, will considerably facilitate the process, reducing time and increasing the level of observer's safety.

\section{ACKNOWLEDGEMENTS}

The research leading to these results has received funding from the Polish-Norwegian Research Programme operated by the National Centre for Research and Development under the Norwegian Financial Mechanism 2009-2014 in the frame of Project Contract No 197810.

\section{REFERENCES}

CEMP standard methods, 2014. http://www.ccamlr.org/en/system/files/CEMP\%20Standard\%20Methods\%20Jun\%202 014.pdf

Chwedorzewska, K.J. and Korczak M. 2010. Human impact upon the environment in the vicinity of Arctowski Station, King George Island, Antarctica. Polish Polar Research 31, pp. 4560.

Hajduk, J. and Rodzewicz, M., 2014. Some optimization criteria of the UAV photogrammetric flight-tracks planning. In: 
The International Archives of the Photogrammetry, Remote Sensing and Spatial Information Sciences, Volume XL-1/W4, 2015 International Conference on Unmanned Aerial Vehicles in Geomatics, 30 Aug-02 Sep 2015, Toronto, Canada

34th Symposium of the European-Association-of RemoteSensing-Laboratories (EARSeL), Warsaw, Poland.

Korczak-Abshire, M., Węgrzyn M., Angiel P. and Lisowska M. 2013. Pygoscelid penguins breeding distribution and population trends at Lions Rump rookery, King George Island. Polish Polar Research, 34 (1), pp. 87-99.

Korczak-Abshire, M., Zmarz, A., Chwedorzewska, K.J., Karlsen, S.R., Rodzewicz, M., Storvold, R., Goraj, Z., 2014. UAV for monitoring of indicator species on Antarctic protected areas. In: 34th Symposium of the European-Association-of Remote-Sensing-Laboratories (EARSeL), Warsaw, Poland.

Solbø, S. and Storvold, R., 2013. The CryoWing unmanned aerial system for scientific measurements. In: First Conference of the International Society for Atmospheric Research using Remotelypiloted Aircraft, ISARRA, Palma de Mallorca, Spain.

Xsens Technologies B.V., 2013. http://www.xsens.com/en/general/mti-g.

Infrared Cameras Inc., 2012. http://www.infraredcamerasinc.com/infrared-camera-7640.html 